\title{
Le haut bassin de l'Ouanne Conséquences des transformations du paysage agraire sur la capacité de stockage de l'eau dans les sols
}

\author{
The high basin of the Ouanne \\ Consequences of transformations of the farmland
}

on the water storage capacity of the soil

\section{O. Baumann}

Docteur de $3^{\mathrm{e}}$ cycle en géographie physique

Résumé du mémoire couronné par le Prix Henri Milon 1984 de la Société Hydrotechnique de France

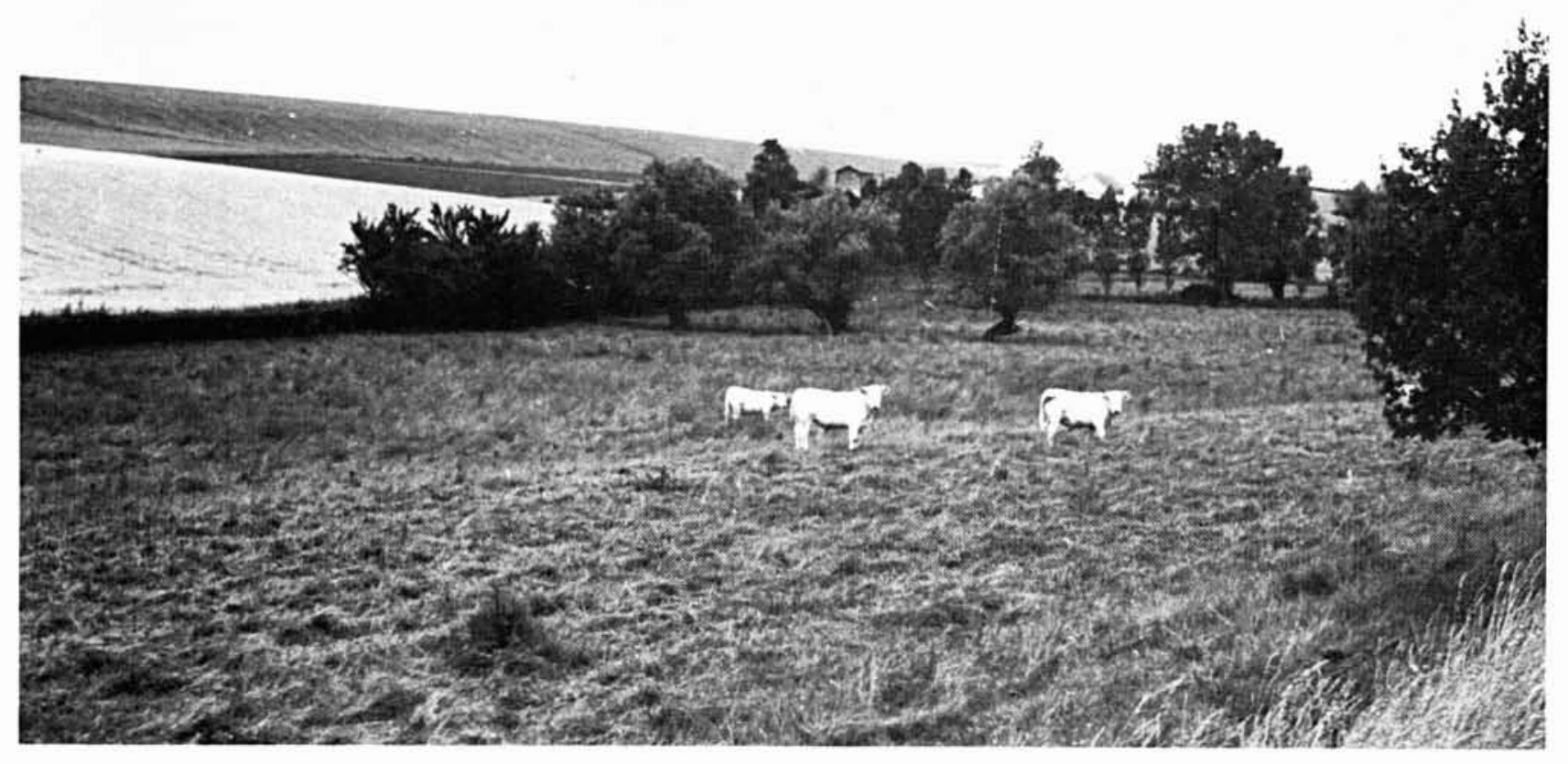

Bocage résiduel de fond de vallée des plateaux jurassiques. Sud de l'Ouanne.

L'auteur donne un aperçu de l'état des connaissances sur le dimensionnement des déversoirs. compte tenu de leur évolution, et analyse: les organes d'entrée, la zone en aval de l'entrée jusqu'à la dissipation d'énergie. la dynamique des jets, les pressions turbulentes dans les bassins de dissipation revêtus, le processus érosif.

Il étudie ensuite. théoriquement et expérimentalement. l'effet du choc entre les jets descendants des déversoirs de surface et le jet ascendant de l'orifice profond (jets croisés). et présente les résultats qui permettent d'évaluer la réduction du potentiel érosif de l'ensemble.
A summary is given of the state-of-the-art on the dimensioning of spillways, having in mind their evolution and analysing: the intake components, the zone upstream of the intake as far as the energy dissipation; the dynamics of the jets turbulent pressures in lined stilling basins; the scour process.

Lastly, a theoretical and experimental study is made on the effect of the shock between the descending jets of the surface spillways and the ascending jets of the hottom jets crossed jets - results being presented that make it possible to estimate the reduction in the scour potential of the whole discharge. 


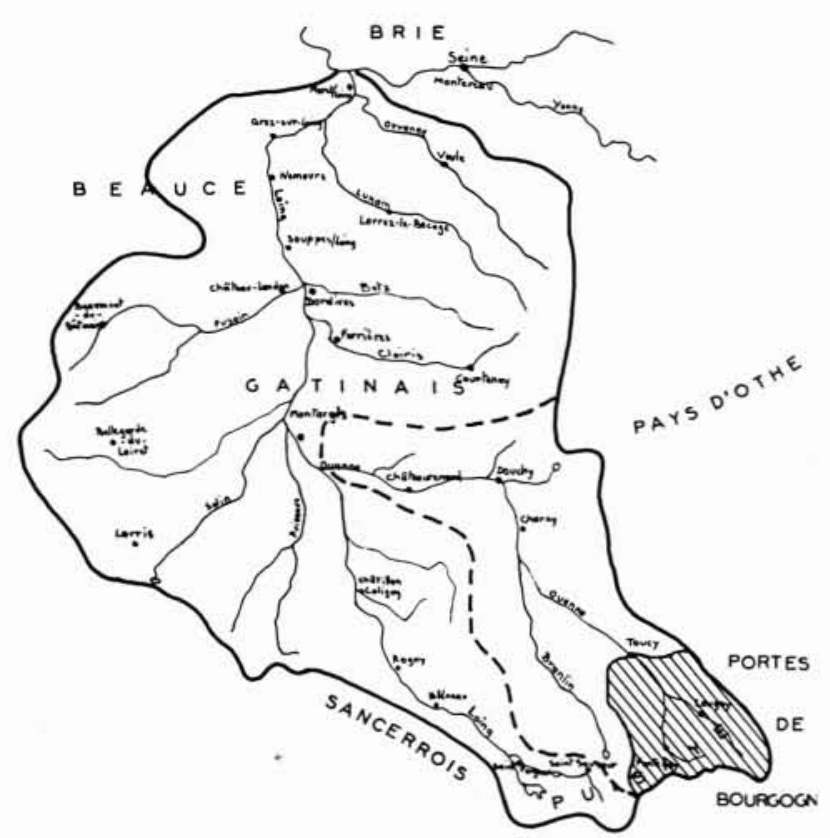

Limites do beseis rereant du boing

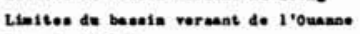
Terrais d'tiode

Figure 1. - Localisation du secteur d'étude (échelle 1: 1200000 )
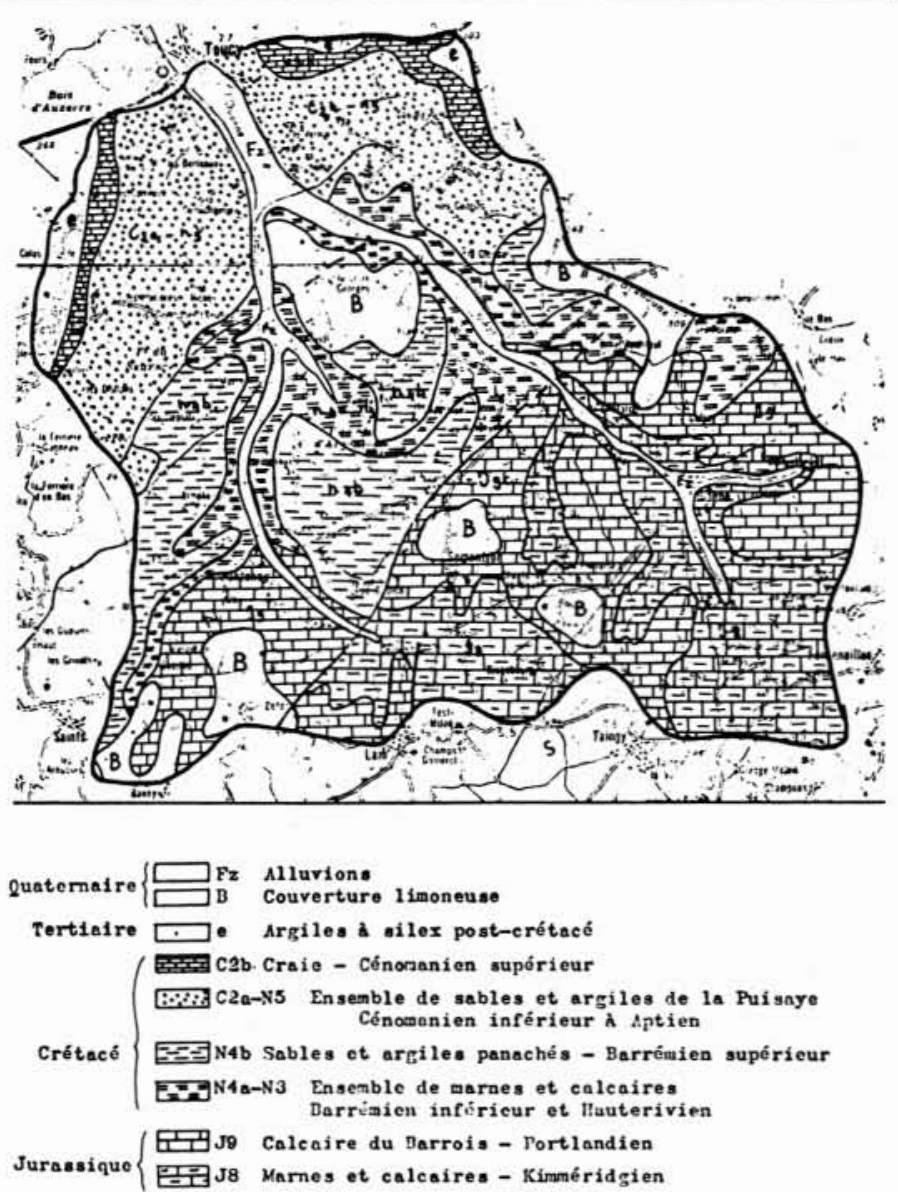

Figure 2. - Extrait des cartes géologiques d'Auxerre et Courson. (échelle 1: 190000 ).

\section{Thème de recherche Méthodologie générale de l'étude}

L'étude se propose d'apporter des éléments de réponse quantitatifs aux questions de la modification du régime des eaux superficielles liée à des transformations agraires.

Elle consiste d'une part à établir une méthodologie capable de répondre à ce problème, et d'autre part, à appliquer cette méthodologie sur un bassin versant affecté par ce type de problème.

La phase d'application a donc un double rôle :

- vérifier le bon fonctionnement de la méthode proposée;

- établir des résultats concrets et quantifiés.

Le bassin versant retenu est celui de l'Ouanne en amont de Toucy, dans l'Yonne. Au cours des dernières décennies, de profondes mutations l'ont affecté : suppression intense des haies de bocage, transfert massif des prairies initiales au profit des cultures céréalières. Aujourd'hui, il connait des pointes de crues de plus en plus fréquentes et subites.

L'étude s'est fixée comme but à atteindre :

a) de définir les ordres de grandeur de la capacité de stockage d'une haie barrant un versant en fonction du type de sol et de l'occupation du sol. (échelle de la parcelle).

b) de définir la variation de la capacité de stockage du bassin versant entre les années 1950 et 1980, liée à la transformation des structures bocagères et à la modification de l'occupation du sol. (échelle du bassin versant).

La méthodologie repose sur les idées suivantes :

Les incertitudes liées aux données pluviométriques, l'impossibilité de mesurer l'évapotranspiration réelle, la méconnaissance des régimes d'infiltration et de circulation profonde des eaux infiltrées, enfin les difficultés de disposer de mesures fiables sur le débit d'un cours d'eau font qu'il est aléatoire d'utiliser les méthodes reposant sur la notion de bilan hydrique pour mettre en évidence le rôle des paramètres jouant sur le transfert de l'eau dans le sol, tels que la modification de structure du sol, les transformations de pratiques culturales, la suppression de structures bocagères.

Si bien que l'on est amené à utiliser une méthode qui s'oppose aux méthodes globales et déductives axées sur l'étude des bilans hydriques : la méthode mise en œuvre dans cette étude ne s'intéresse pas aux transferts ou modifications de transfert de l'eau au niveau du bassin versant; au contraire, et c'est là son originalité, elle s'intéresse au rôle de stockage au niveau de la haie barrant une séquence bocagère. Savoir comment l'eau est retenue au niveau de la haie barrant un versant, et quel volume est mis en jeu, constituent donc la notion centrale de l'étude.

Ce n'est qu'une fois ce rôle de stockage défini, et muni de résultats quantitatifs du stockage représentatifs des principaux domaines pédologiques du bassin versant, que l'on envisage l'approche à l'échelle du bassin versant en utilisant à la fois les résultats d'expérimentations à l'échelle de la parcelle, et à la fois les données relatives aux transformations agraires survenues sur le bassin versant entre les années 1950 et 1980. 

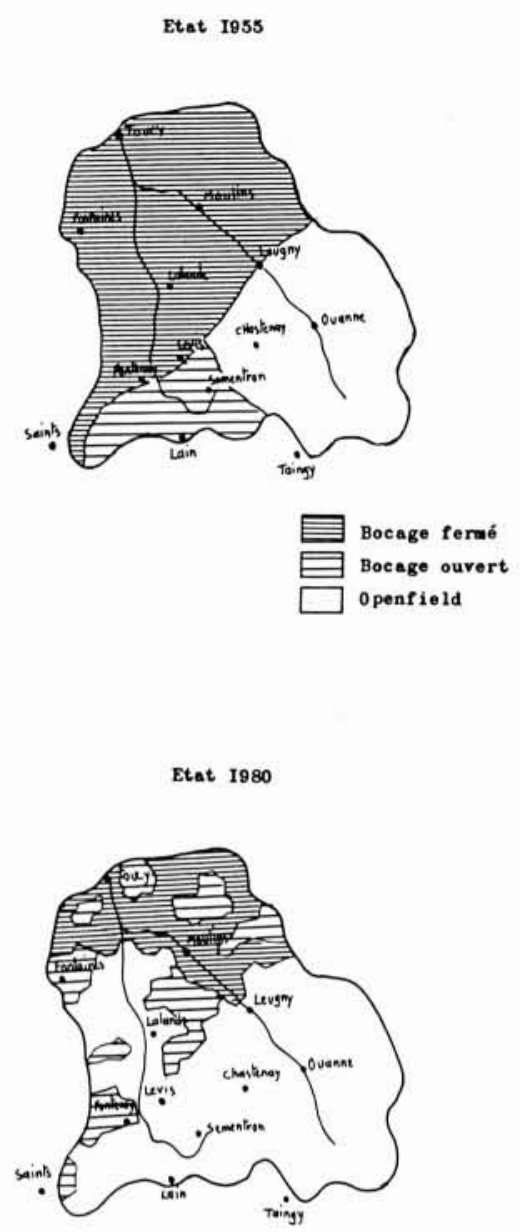

Figure 3. - Évolution des structures paysagères : 1955-1980.

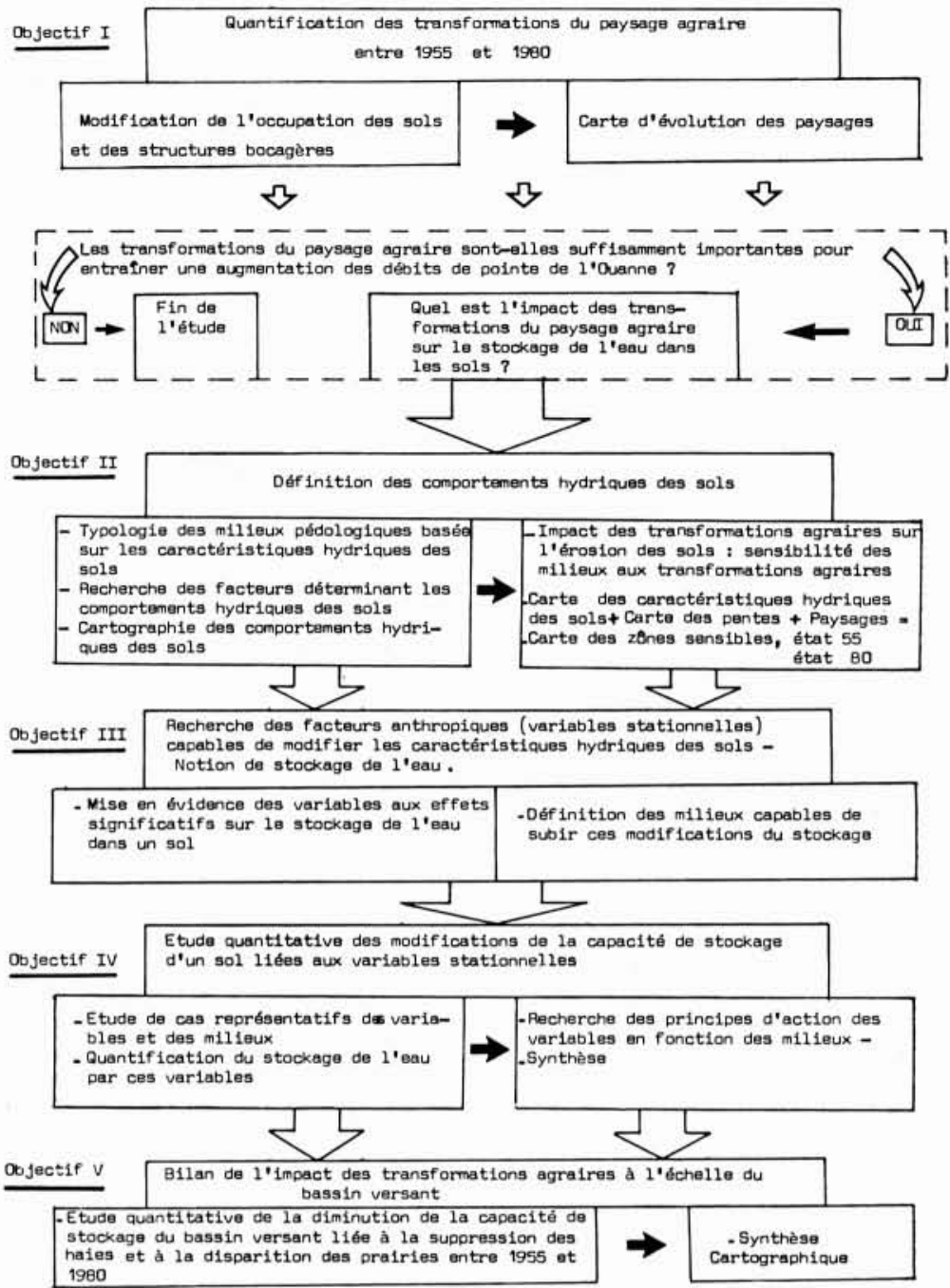

Figure 4. - Organigramme de la démarche générale.
Cette méthode a donc comme souci constant de n'utiliser que des données mesurées in situ en laissant le moins de place possible aux données issues d'extrapolations, ou de déductions théoriques.

\section{Démarche de l'étude \\ Moyens mis en œuvre. Principaux résultats}

La démarche de l'étude consiste à résoudre successivement cinq objectifs principaux, les résultats de chacun d'eux étant réinvestis en vue de la résolution de l'objectif ou des objectifs suivants. La figure 4 donne l'organigramme de la démarche générale.

On s'attachera donc à présenter ces différents objectifs ainsi que leurs principaux résultats.

\section{Etude quantitative de la dynamique des paysages agraires}

Deux maquettes de photo-interprétation représentent l'état du bassin versant en 1955 et en 1978. L'application sur ces maquettes de grilles de comptage par maille permet un dénombrement du kilométrage de haies, et des surfaces d'utilisation du sol.

L'évolution des structures des paysages agraires a été la suivante :

- sur $1950 \mathrm{~km}$ de haies en $1955,1200 \mathrm{~km}$ ont été supprimés. La densité moyenne du bocage sur le bassin versant est passée ainsi de $125 \mathrm{~m}$ de haie par hectare en 1955, à $53 \mathrm{~m}$ actuellement;

- sur 5700 hectares de prairies en 1955, 1800 ont été supprimés, alors que dans le même temps les surfaces cultivées ont augmenté de 1700 hectares;

- enfin, les surfaces boisées ont légèrement augmenté, passant de 2250 à 2400 hectares.

Une cartographie détaillée traduit cette évolution. 
Tableau I. - Évolution de I'occupation du sol et des structures bocagères sur le bassin versant entre 1955 et 1978.

Tableau II. - Synthèse des comportements hydriques des sols.

Favorise tres forteent 10 ruiesellemen Favorise fortement 10 raiesellemt Pavoriae moyenneent 10 ruiseollement S'oppere au ruisuellement

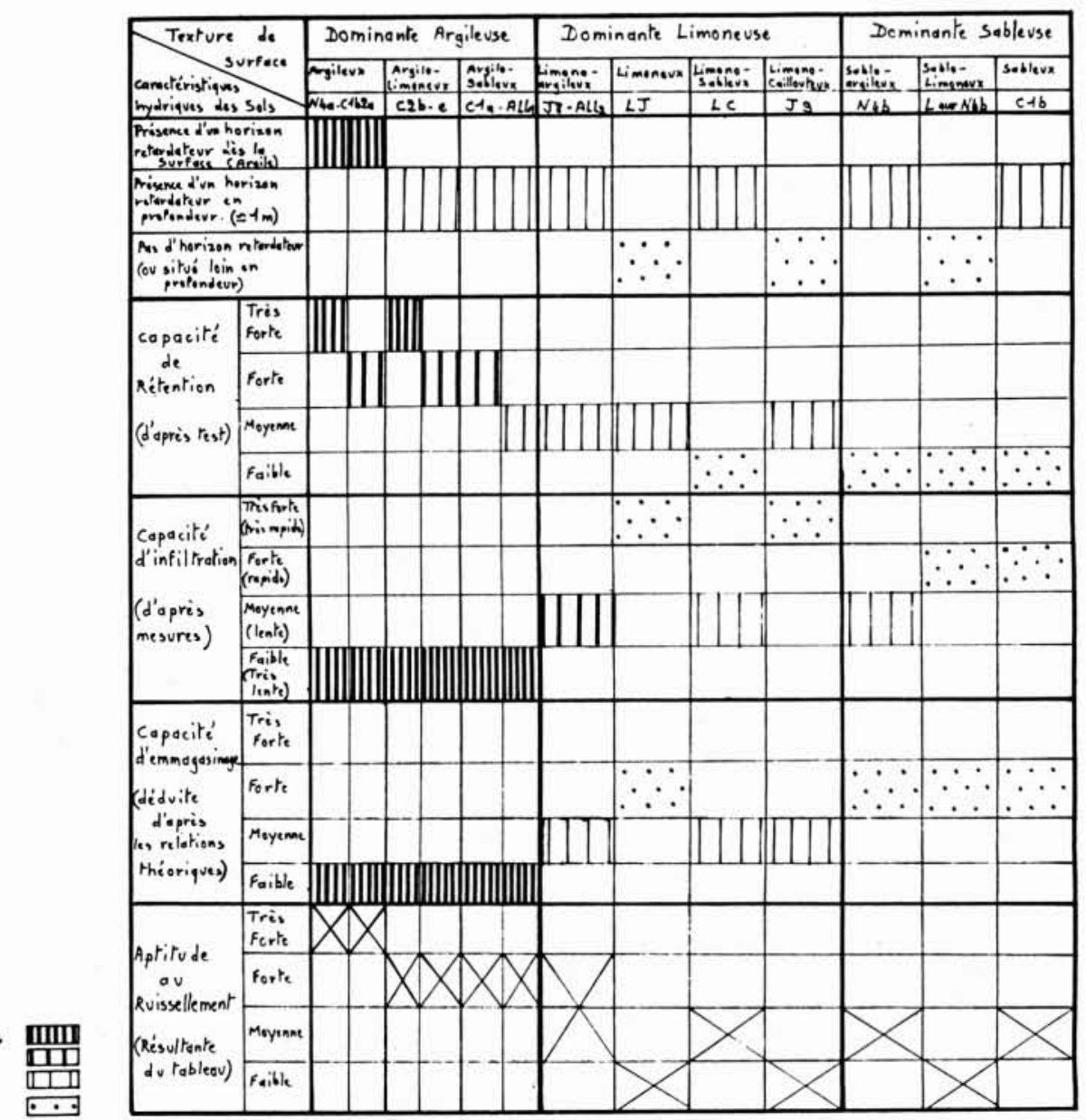

\begin{tabular}{|c|c|c|c|}
\hline & 1955 & 1978 & Évolution \\
\hline $\begin{array}{l}\text { réseau } \\
\text { des haies } \\
\text { prairies } \\
\text { cultures } \\
\text { bois }\end{array}$ & $\begin{array}{c}1950 \mathrm{~km} \\
5700 \text { ha }(36 \%) \\
7500 \text { ha }(47 \%) \\
2250 \text { ha }(14 \%)\end{array}$ & $\begin{array}{c}825 \mathrm{~km} \\
3900 \text { ha }(24 \%) \\
9200 \text { ha }(58 \%) \\
2400 \text { ha }(15 \%)\end{array}$ & $\begin{array}{l}-1100 \mathrm{~km} \\
-1800 \text { ha }(-12 \%) \\
+1700 \text { ha }(+11 \%) \\
+\quad 150 \text { ha }(+1 \%)\end{array}$ \\
\hline
\end{tabular}

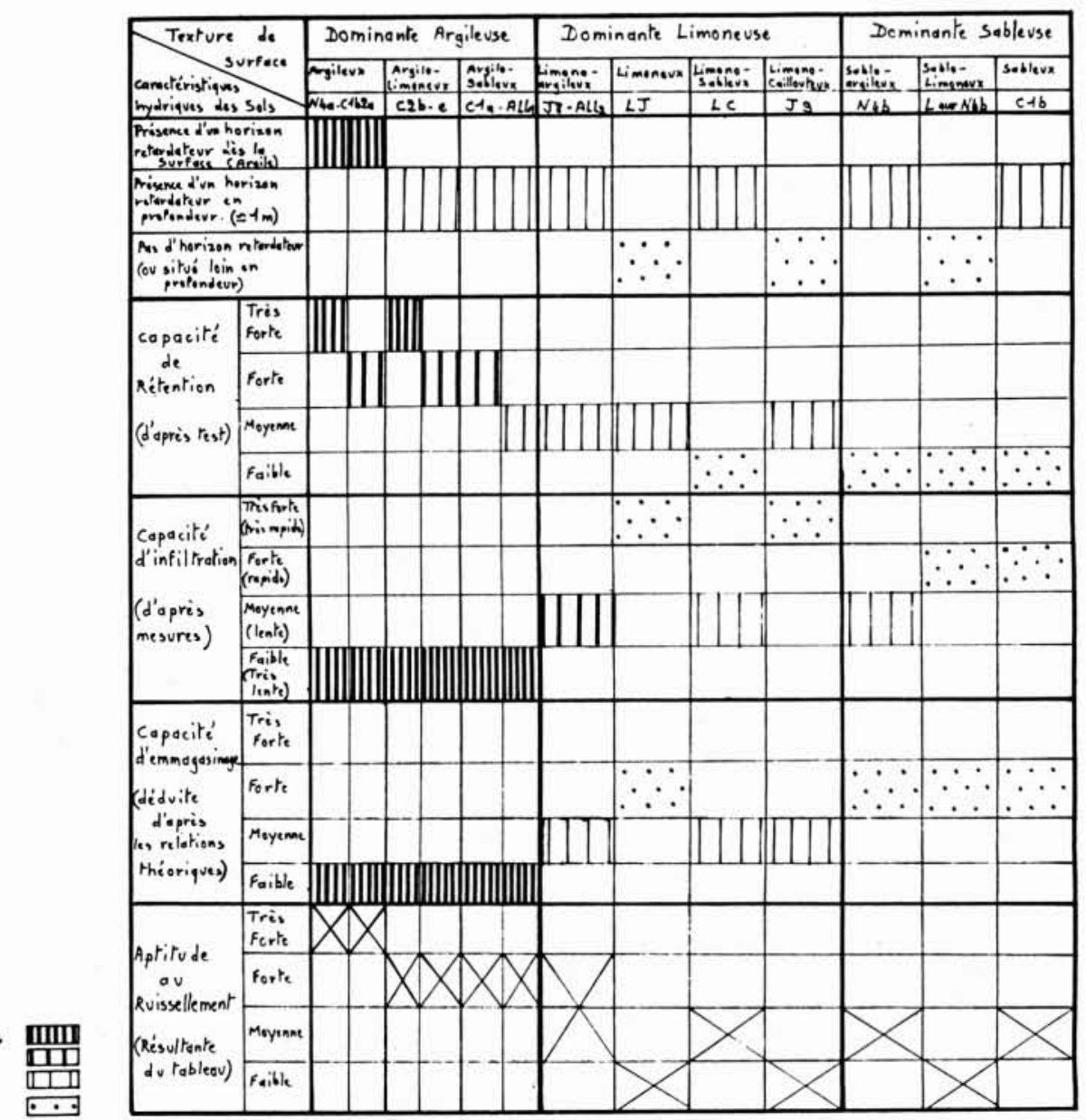

\begin{tabular}{|c|c|c|c|c|c|}
\hline & $\begin{array}{c}\text { Variables } \\
\text { stationnelles }\end{array}$ & $\begin{array}{l}\text { Sables } \\
5 \%\end{array}$ & $\begin{array}{c}\text { argile } \\
10-12 \%\end{array}$ & $\begin{array}{c}\text { Limons } \\
20-30 \% \text { d'argile } \\
\end{array}$ & $\begin{aligned} & \text { Argiles } \\
&> 30 \% \text { argile } \\
&\end{aligned}$ \\
\hline & Type de haie & faible & & & très forte \\
\hline$\stackrel{\Xi}{\partial}$ & Utilisation du sol & faible & faible & forte & très forte \\
\hline 흔 & Tassement du sol & faible & & & forte \\
\hline 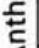 & Etat de surface & & faible & & forte \\
\hline$\ll$ & Façons culturales & & faible & forte & faible \\
\hline$\stackrel{\mathscr{g}}{=}$ & Position topographique & & & & \\
\hline$\frac{\pi}{w}$ & Pente & & & & \\
\hline$\frac{\varepsilon}{\alpha}$ & Exposition & faible & & & \\
\hline
\end{tabular}

Tableau III. - Influence des variables stationnelles sur I'humidité des sols. 
Etude hydro-pédologique : comportements hydrique des sols

Il s'agit de rechercher les facteurs responsables des différenciations hydriques des sols, de définir leurs comportements hydriques et d'aborder, à partir de cette notion de comportement hydrique, la question de la sensibilité des milieux pédologiques aux transformations agraires. On sait en effet que l'érosion d'un sol dépend en grande partie de son aptitude à ruisseler, elle même dépendante des paramètres hydrodynamiques (capacités d'infiltration, d'emmagasinage, pente,) et anthropiques (présence de haies barrant les versants, taux de recouvrement du sol par les cultures,.).

On a donc tenté de définir les sols les plus sensibles à l'érosion en recherchant les facteurs physiques et anthropiques qui étaient capables d'entraîner des comportements hydriques spécifiques.

Des expériences de vitesse d'infiltration in situ, des tests de rétention, des analyses granulométriques et sédimentologiques ont permis de définir les grands domaines pédologiques du bassin versant et leurs comportements vis-à-vis de la dynamique de l'eau dans les sols.

Lors de ces expériences sur le terrain, des relevés méthodiques des différents milieux, effectués simultanément, ont permis de mener une analyse factorielle du comportement hydrique des sols de Puisaye.

Le tableau II donne les résultats de cette analyse, qui a également servi à dresser une cartographie des comportements hydriques des sols.

\section{Recherche des facteurs anthropiques capables de modi- fier les comportements hydriques des sols}

Connaissant les caractéristiques hydriques des sols et leur comportement général vis-à-vis de l'emmagasinage de l'eau, on a ensuite recherché l'existence de facteurs anthropiques capables de modifier les possibilités de stockage de l'eau dans les sols, ainsi que les conditions de leur action.

La capacité de stockage de l'eau dans un sol se définit comme étant l'aptitude du sol à conserver l'eau dans les horizons supérieurs, en relation avec les variables d'état du milieu. La capacité de stockage dépend donc des caractéristiques hydriques propres au sol (rétention, emmagasinage,.), et des caractéristiques de variabilité propres au milieu (occupation du sol, présence de haies, pente, exposition, façons culturales,.).

Des mesures d'humidité de surface ont été effectuées sur l'ensemble du bassin versant en même temps qu'un relevé systématique de milieu aux environs de la station de mesure.

La recherche de corrélations, par type textural de sol, entre les variables spécifiques du milieu (exposition, pente, topographie, utilisation du sol, présence de haies, tassement des structures, etc.) et des mesures d'humidité de surface effectuées sur l'ensemble du bassin versant, a mis en évidence des " anomalies" d'humidité pour certains types de sols, liées à la présence de certains facteurs :

Les relations se sont révélées les suivantes :

- Plus le milieu est riche en argile (seuil significatif à partir de $30 \%$ ), plus les écarts d'humidité sont forts pour un même type de sol;

- plus le milieu est riche en sable, et pauvre en argile, plus les écarts d'humidité sont faibles pour un même type de sol;

- lorsque le milieu est pauvre en argile, la capacité de stockage du sol n'est pas susceptible de varier profondément selon l'état stationnel du milieu. Elle dépend seulement des caractéristiques hydriques propres au sol;

- au contraire, lorsque le milieu est riche en argile $(30 \%$ et plus), la capacité de stockage du sol est susceptible de varier profondément, ne dépendant pas seulement des caractéristiques hydriques du sol (capacité d'infiltration, de rétention, d'emmagasinage), mais également de l'état stationnel du sol (utilisation du sol, tassement des structures, pourcentage de recouvrement par la végétation, présence des haies);

- les variables les plus significatives, pour cette question des modifications de la capacité de stockage d'un sol, sont regroupées dans le tableau ci-dessous;

- la présence des haies barrant les versants, le type d'occupation du sol opposant prairies et labours prennent une importance particulière.

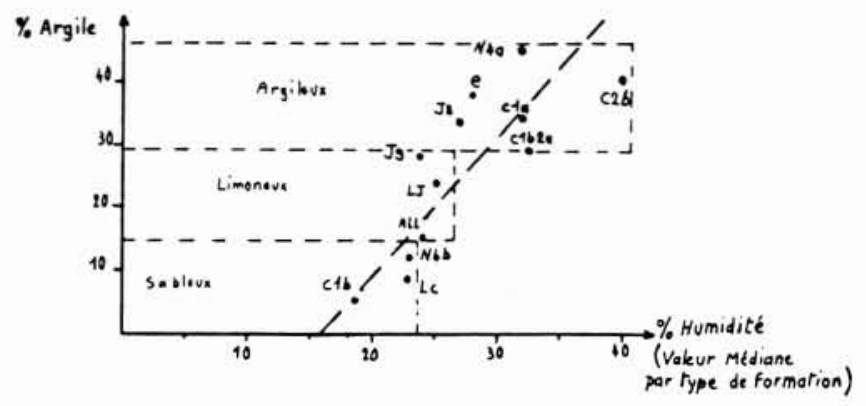

Figure 5. - Relation entre l'humidité (valeur médiane) et le pourcentage d'argile de surface.

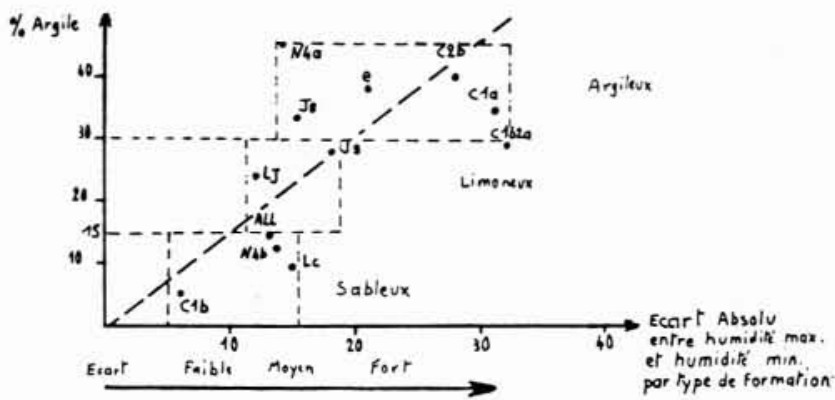

Figure 6. - Relation entre le pourcentage d'argile et l'écart absolu des valeurs d'humidité, par type de substrat. 


\section{Étude quantitative du stockage de l'eau par les systèmes barrant les versants}

En comparant les teneurs en eau des sols en surface et en profondeur sur des parcelles juxtaposées comparables en tous points (exposition, pente, sol, substrat...) à l'exception d'un caractère anthropique (présence ou absence de haie barrant le versant, utilisation du sol), le phénomène de stockage a pu être mis en évidence.

Du point de vue qualitatif, les résultats suivants peuvent être avancés :

- l'essentiel des variations d'humidité liées à la présence d'une haie barrant un versant se produit dans les 50 premiers centimètres de sol, et ne semble pas affecter (ou peu) la profondeur;

- la haie et (ou) le talus jouent un rôle d'obstacle mécanique à l'écoulement latéral de l'eau dans les horizons supérieurs du sol. Mais il existe une différence de comportement selon qu'il s'agit d'une haie sur talus, ou seulement d'un talus (haie supprimée) : dans le premier cas on note une augmentation d'humidité dans le talus (système racinaire de la haie) en amont du talus, et sur les $2 / 3$ de la parcelle, en partie haute. Dans le second cas, on note une augmentation d'humidité seulement juste en amont du talus.

Afin d'évaluer l'ordre de grandeur du stockage de l'eau par les haies on a comparé, sur des transects d'humidité effectués sur les différents couples de parcelles étudiées, la réserve hydrique du sol (Formule de Hénin), en exprimant les résultats en mètres cubes par mètre linéaire de haie.

Différents types pédologiques, et différentes conditions d'utilisation du sol ont ainsi été étudiés.

Les résultats figurent dans le tableau $I V$.

Une parcelle labourée, même barrée par une haie, a une capacité de stockage deux fois plus faible qu'une parcelle en prairie barrée par une haie.

Le type de sol intervient de façon significative sur le stockage. Plus le milieu est argileux, plus le rôle des haies barrant les versants est important. Plus le milieu est sableux, plus ce rôle est modeste. En considérant deux prairies, l'effet de stockage sur sol argileux est deux fois plus efficace que sur sol sableux $\left(7,5 \mathrm{~m}^{3}\right.$ contre $\left.3 \mathrm{~m}^{3}\right)$.

Le type d'occupation du sol est lui aussi un facteur de première importance. Les milieux peu ou non travaillés à recouvrement très denses s'opposent aux milieux fortement travaillés à recouvrement faible ou nul (labours) : là encore le rapport est de deux pour un.

Les schémas de la figure 7 montrent les relations établies.

Étude quantitative de la variation de la capacité de stockage par les structures bocagères à l'échelle du bassin versant entre 1950 et 1980.

L'extrapolation des résultats de l'étude stationnelle sur le stockage par les haies barrant les versants a pu être réalisée à l'échelle du bassin versant pour les années 1955 et 1978 à partir des maquettes des paysages agraires, qui permettent pour chaque secteur de calculer la longueur des haies barrant les versants, et la superficie des terres en prairies et en labours, ainsi qu'à partir de la carte des comportements hydriques des sols, qui permet un découpage du bassin en secteurs pédologiques ayant des comportements homogènes vis-à-vis du stockage.

Le tableau $V$ donne les résultats de l'étude.

La capacité de stockage en 1978 est réduite de plus de la moitié par rapport à celle de 1955. 3,8 millions de $\mathrm{m}^{3}$ pouvaient être stockés en 1955 , seulement 1,7 millions de $\mathrm{m}^{3}$ peuvent être stockés en 1978. La différence est estimée à 2 millions de $\mathrm{m}^{3}$.

Une traduction cartographique de ces résultats a été réalisée destinée à visualiser les secteurs du bassin les plus perturbateurs. A partir de cette cartographie, et comptetenu de l'évolution des structures du bassin versant, des propositions d'aménagement destinées à laminer les pointes de crues à l'exutoire ont été formulées. Ces propositions d'aménagement reposent sur l'idée qu'il faudrait réaugmenter la capacité de stockage du bassin versant afin de retrouver des conditions d'écoulement se rapprochant de celles qui existaient lorsque le bocage était encore présent.

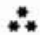

Les moyens mis en œuvre dans cette étude ont permis de dégager quelques principes nouveaux sur l'hydrologie des séquences bocagères, par un éclairage nouveau de la question des conséquences des transformations des structures bocagères à l'aide du concept de stockage de l'eau dans les sols.

Un axe de recherche se trouve tracé dans le prolongement de cette étude : dépasser l'aspect statique de la notion de stockage pour parvenir à une approche dynamique, l'étude des transferts d'eau sur séquences bocagères et de leur modification par la suppression des systèmes barrant les versants.
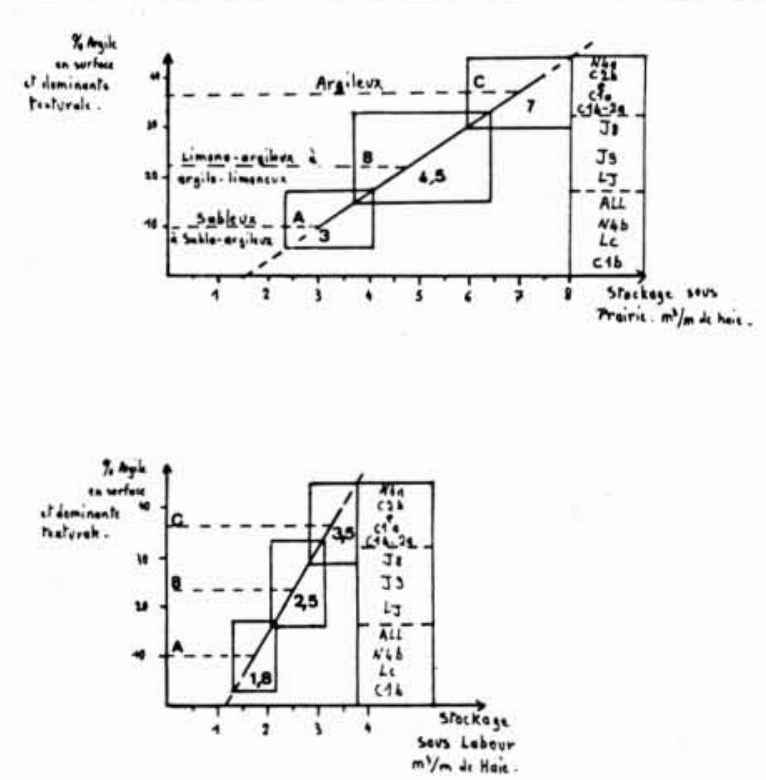

Figure 7. - Relation entre le type sédimentologique de milieu et l'aptitude au stockage de l'eau par les haies pour les prairies et les labours. 


\begin{tabular}{|c|c|c|c|c|c|c|c|}
\hline \multirow{2}{*}{$\begin{array}{c}\text { oubatrat } \\
\begin{array}{c}\text { argile } \\
\text { et } \\
\text { arnes }\end{array}\end{array}$} & \multicolumn{3}{|c|}{$\begin{array}{l}\text { caracteres } \\
\text { lithologiques de surface } \\
\text { Sargilo-xilimon-s,auble }\end{array}$} & \multirow{2}{*}{$\begin{array}{c}\begin{array}{c}\text { oceupation } \\
\text { du } \bullet 01\end{array} \\
\begin{array}{c}\text { prairie } \\
\text { amont/oral }\end{array}\end{array}$} & \multirow{2}{*}{\begin{tabular}{|c|} 
pente \\
10 : \\
$12 *$
\end{tabular}} & \multirow{2}{*}{\begin{tabular}{|c|} 
ayotidee barrant \\
10 veraent \\
$\begin{array}{c}\text { haie our } \\
\text { talue }\end{array}$
\end{tabular}} & \multirow{2}{*}{$\begin{array}{l}\text { otockage } \\
\text { mo de haie } \\
\text { haie et } \\
\text { taluat } 0,2 \\
\text { - amont: } 0,5 \\
\text {-induit } 7 \\
\text {-TOTAL: } 7,5 \\
\end{array}$} \\
\hline & 418 & $42 \pi$ & 178 & & & & \\
\hline $\begin{array}{l}\text { orgile } \\
\text { et } \\
\text { marnes }\end{array}$ & 418 & $42 x$ & $7 \pi$ & $\begin{array}{c}\text { prairie } \\
\text { amont/aral }\end{array}$ & ${ }_{12}^{10}$ & (talua arracbée) & 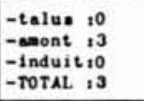 \\
\hline $\begin{array}{l}\text { abble } \\
\text { argileux }\end{array}$ & ${ }_{10 \%}^{58}$ & $\begin{array}{l}58 \\
10 \%\end{array}$ & $\begin{array}{l}80 \% \\
90 \%\end{array}$ & $\begin{array}{c}\text { prairse } \\
\text { amont/aral }\end{array}$ & $10^{\circ}$ & $\begin{array}{l}\text { haie our } \\
\text { talue }\end{array}$ & $\begin{array}{l}\text { haie et } \\
\text { talua :0,25 } \\
\text {-induiti2,5 } \\
\text {-TOTAL 13 }\end{array}$ \\
\hline $\begin{array}{l}\text { abble } \\
\text { argileux }\end{array}$ & $\begin{array}{l}5 \mathrm{a} \\
10 \pi\end{array}$ & $\begin{array}{l}58 \\
10 \%\end{array}$ & ${ }_{90 \pi^{8}}^{80}$ & $\begin{array}{c}\text { 1abour } \\
\text { amont/aval }\end{array}$ & ${ }_{8}^{\circ}$ a & $\begin{array}{l}\text { haie our } \\
\text { telue }\end{array}$ & $\begin{array}{l}\text { baie et } \\
\text { talus i0,2 } \\
\text {-induit, I, 25 } \\
\text {-TOTAL iI,5 }\end{array}$ \\
\hline $\begin{array}{l}\text { abble } \\
\text { argileux }\end{array}$ & $\begin{array}{l}51 \\
105\end{array}$ & $\begin{array}{l}5 \mathrm{~A} \\
10 \%\end{array}$ & ${ }_{90 \pi}^{80}$ & labour & $10^{\circ}$ & (haie "rien" & -0 \\
\hline
\end{tabular}

Tableau IV. - Stockage de l'eau par les haies.

\begin{tabular}{|c|c|c|c|c|c|c|c|}
\hline \multirow[b]{2}{*}{ Bnes } & \multicolumn{3}{|c|}{ Structures bocagires 195S } & \multicolumn{3}{|c|}{ Structures bocagères 1978} & \multirow[b]{2}{*}{$\begin{array}{l}\text { différence } \\
\text { 1955- } \$ 979\end{array}$} \\
\hline & $\begin{array}{l}\text { Stockage } \\
\text { prairie }\end{array}$ & $\begin{array}{l}\text { stockage } \\
\text { labour }\end{array}$ & $\begin{array}{l}\text { stockagu } \\
\text { total }\end{array}$ & \begin{tabular}{|l|} 
stodkage \\
erairie
\end{tabular} & $\begin{array}{l}\text { Stockaga } \\
\text { labour }\end{array}$ & $\begin{array}{l}\text { stockage } \\
\text { total }\end{array}$ & \\
\hline Argilleuse & 1410700 & 414800 & 1825000 & 512400 & 313200 & exso00 & -1000000 \\
\hline Argillo-sablouse & a65200 & 5200 & 270000 & 169500 & 21100 & 190000 & -80000 \\
\hline Limono-argileuse & 90000 & 180000 & 279000 & $\Delta 0500$ & 45300 & 86000 & -193000 \\
\hline Limoneuse & 297200 & 446400 & 744000 & 300700 & 113400 & 414000 & -330000 \\
\hline Sableuse & 519400 & 166500 & 606000 & 154800 & 100000 & 264000 & - 577000 \\
\hline Total & 2502000 & 1222000 & 3000000 & 1777000 & 6c2000 & 1780000 & -2000000 \\
\hline
\end{tabular}

Tableau V. - Estimation des volumes d'eau retenus par les haies en 1955 et en 1978 (les volumes sont exprimés en $\mathrm{m}^{3}$ ).

\section{Orientation bibliographique}

\begin{tabular}{|c|c|}
\hline $\begin{array}{c}\text { CARNET (C.) } \\
1978\end{array}$ & $\begin{array}{l}\text { Étude des sols et de leur régime hydrique } \\
\text { en région granitique de Bretagne: une } \\
\text { approche du róle du bocage - Thèse de } \\
3^{e} \text { cycle - INRA - ENSA - Université } \\
\text { de Rennes. }\end{array}$ \\
\hline $\begin{array}{c}\text { BAUMANN (O.) } \\
1983\end{array}$ & $\begin{array}{l}\text { Le Haut Bassin de l'Ouanne - Approche } \\
\text { d'un bocage en mutation - Conséquences } \\
\text { des transformations du paysage agraire } \\
\text { sur la capacité de stockage de l'eau dans } \\
\text { les sols - Thèse } 3^{e} \text { cycle - Université de } \\
\text { paris VII - } 418 \text { p. }\end{array}$ \\
\hline
\end{tabular}

\author{
MÉRoT (Ph.)

MÉrot (Ph.),

BOURGUET (M.)

Le LEUCH (M.),

PÉpIN (D.),

GAREL (C.)

1981
Le bocage en Bretagne granitique: une approche de la circulation des eaux Thèse de $3^{e}$ cycle - Université de Rennes, $196 \mathrm{p}$

Fonctionnement hydrologique de bassins versants élémentaires en Bretagne: rôle des organisations pédologiques - Contrat Ministère de la culture et de l'environnement $\mathrm{n}^{\circ} 77 / 87$ - INRA-ENSA, $167 \mathrm{p}$. 


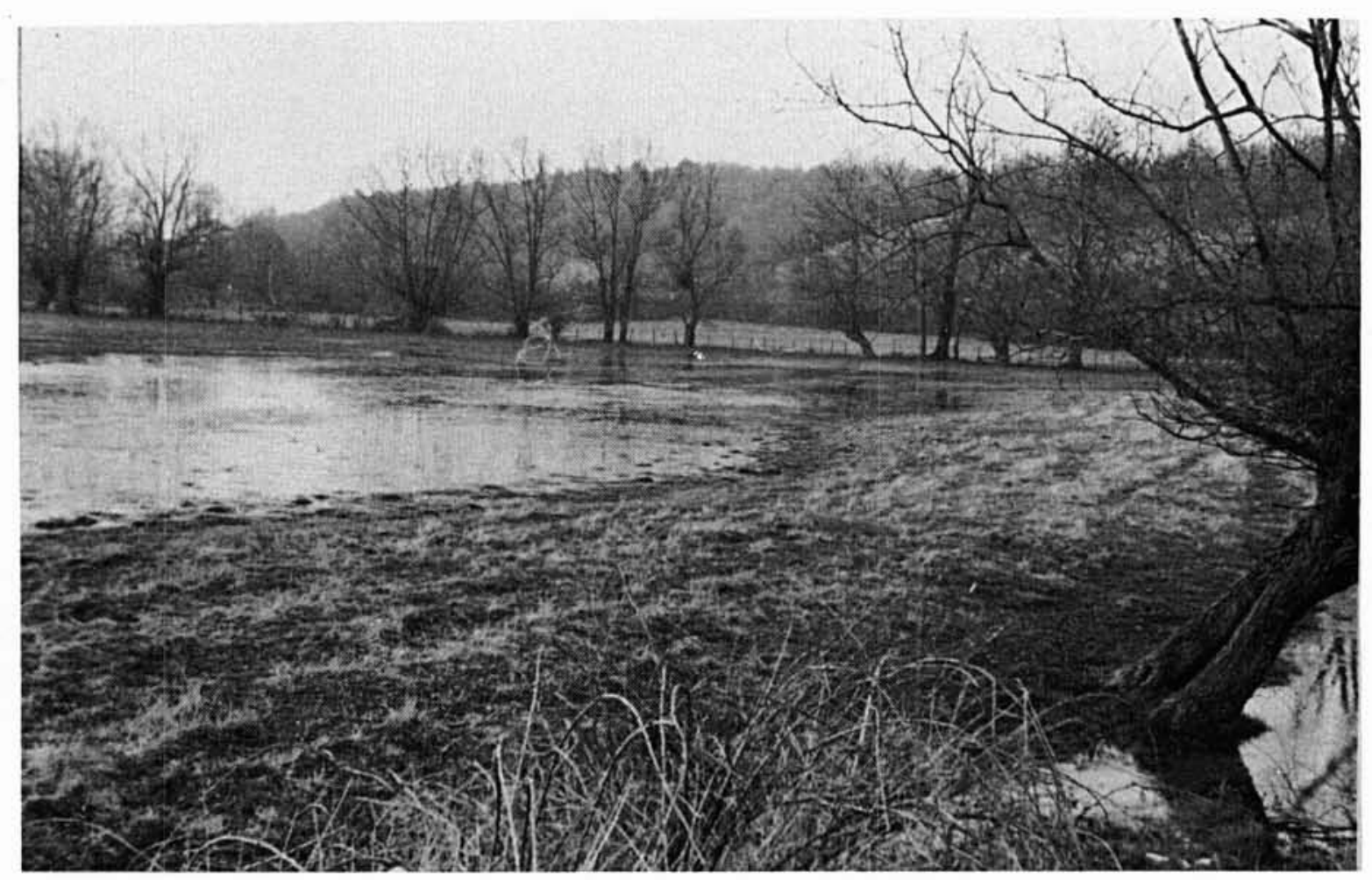

Vallée de l'Ouanne au Moulin Berthier. 